\title{
Mechanical Losses in the Piston-Bushing Contact of Axial Piston Units*
}

\author{
Nils VATHEUER ${ }^{* *}$, Hubertus MURRENHOFF**, \\ Ulrich BRÄCKELMANN ${ }^{* * *}$, David BREUER ${ }^{* * *}$
}

\begin{abstract}
In hydraulic systems with pressure levels above $21 \mathrm{MPa}$, as being typical in mobile applications, piston units are the first choice. Especially axial piston pumps in swash plate design offer technical benefits and enable compact and efficient designs. Worth mentioning here as an example is the power take off (PTO) and the efficient controllability by use of variable displacements. Nevertheless, the tribological contacts in these robust and widely used components are highly stressed and determine the efficiency of the units. Due to the kinematics each piston is highly loaded by lateral forces and therefore the piston-bushing contact represents the area where the tribological knowledge is essential for wear-resistant and efficient design. Besides materials, macro and micro geometry, local loads and movement lead to friction losses. This paper describes the theoretical kinematics of the inclined piston and a sensor for measuring the rotation of the piston in the pressurised piston-bushing contact, which enables to fill the knowledge gap regarding the rotational movement of the piston under operational conditions.
\end{abstract}

Keywords: Hydraulics, Pump, Motor, Tribology, Measurement, Friction, Sensor, Experimental Analysis

\section{Introduction}

Hydrostatic pumps and motors in axial piston design are favourable for many applications with high demands on efficiency and controllability. The detailed investigation of such machines has been in progress since the middle of the past century. The main needs for research to date are the different tribological contacts. Beside the cylinder blockvalve plate contact and the slipper-swash plate contact the piston-bushing contact is highly stressed. One of the first detailed researches on an entire axial piston unit was done by Renius $^{1), 2)}$. Renius found that the piston rotation depends on the operation mode. For the very slow sneaky mode in motor operation (ca. $0.1 \mathrm{rpm}$ ) a slippage up to $78 \%$ is possible. Also in the pump mode at higher speeds $(1000-1500 \mathrm{rpm})$ Renius detected slippage of the piston relative to the drive speed. Fang and Shirakashi described in ${ }^{3)}$ that the piston rotates around its own axis with drive speed under typical conditions. Bräckelmann ${ }^{4)}$ measured the reaction behaviour in the ball joint of the piston-slipper contact. In combination with the friction torque of the piston-bushing contact this behaviour determines the rotation of the piston around its own axis. Breuer ${ }^{5)}$ formulated an equation to calculate the piston rotation as a function of friction torque and forces and

\footnotetext{
* $\quad$ Manuscript received December 10, 2014

** Institute for Fluid Power Drives and Controls - IFAS

Faculty of Engineering, RWTH Aachen University

(Steinbachstraße 53, 52074 Aachen, Germany)

E-mail: Nils.Vatheuer@ifas.rwth-aachen.de

**** Advanced Engineering Pumps and Motors

Bosch Rexroth AG, Germany

(Glockeraustrasse 4, 89275 Elchingen, Germany)
}

piston inertia.

Recapitulating the above mentioned references, it can be said that the piston rotation is desirable for a better hydrodynamic pressure build up in the tribological contact.

The dominating effect for the rotation is the stiffened ball joint to the slipper. So, the piston rotation is usually assumed as full turning without a considerable amount of slippage.

However, the research on the behaviour so far focused on the uninclined piston, which axis is parallel to the drive axis. Therefore this paper describes the theoretical rotational motion due to the kinematics of the piston, which is inclined to the drive axis. The second part presents a sensor, which allows a detailed investigation of the piston rotation in the bushing under realistic conditions.

\section{Nomenclature}

$A$

$B$

$n$

$P$

$p_{p}$

$R$

$S_{\text {swash }}$

$s_{p}$

$s_{1}, s_{2}$

$t$

$v_{p}$

X

$x_{0}, y_{0}, z_{0}$

$x_{p}, y_{p}, z_{p}$

$x_{s}, y_{s}, z_{s}$ matrix of variable vectors

matrix of result vector

drive speed

piston diameter

vector of the ball joint centres

piston pitch radius

plane of the swash plate

stroke of the piston

direction vectors of swash plate

time

position vector of piston

matrix of control variables

global coordinate system

piston coordinate system

slipper coordinate system 


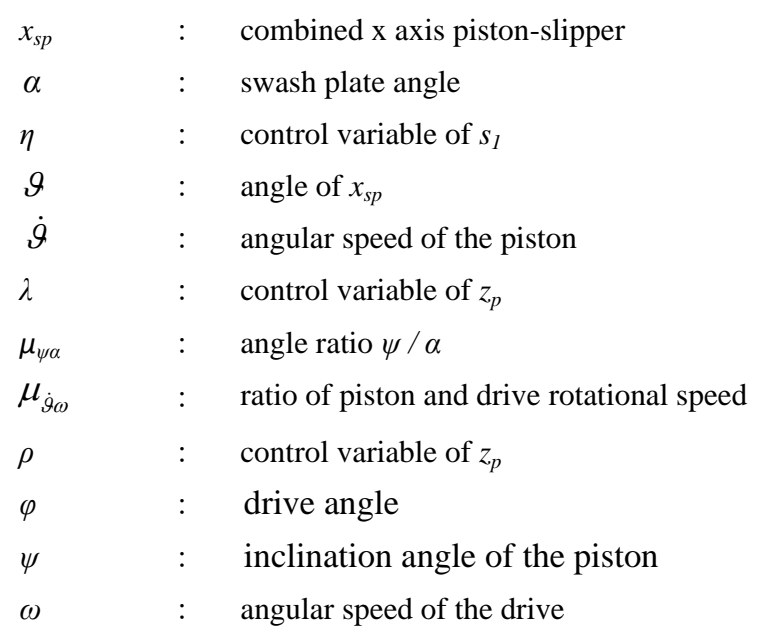

\section{Kinematic Description}

For the theoretical description of the inclined piston kinematics the coordinate system in Fig. 1 is used. The cylinder block rotates around the axis $z_{0}$ with the angle $\varphi$ starting from the axis $x_{0}$ to $y_{0}$. The $x_{0}-y_{0}$ plane is defined by the centres of the ball joints of the pistons by zero-stroke (red line in Fig. 1). The swash plate is swivelling around $x_{0}$ with the angle $\alpha$ counted from $y_{0}$ to $z_{0}$. The inclination of the piston regarding the axis $z_{0}$ is described by the angle $\psi$. The piston pitch radius $R$ is set to the radius of the centre of the ball joint at the angle $\varphi=0$ in the $x_{0}-y_{0}$ plane. This radius is equal to the red line radius. Therefore, the pitch radius is independent from the piston inclination and the swash plate angle.

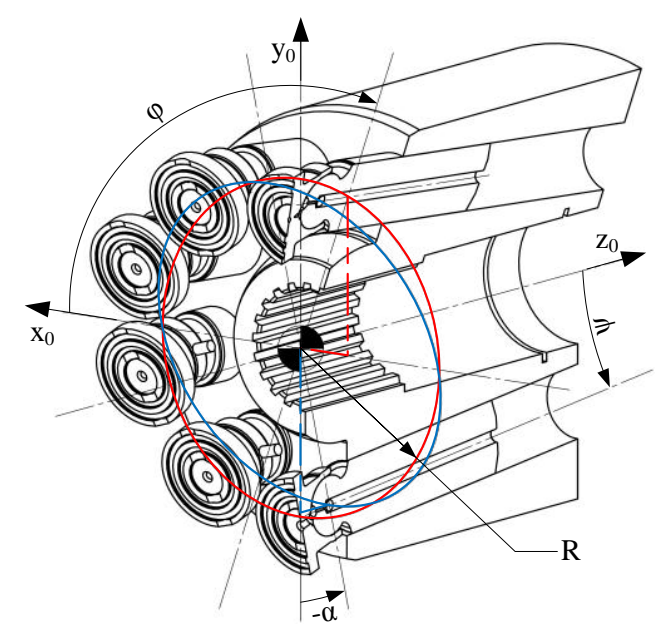

Fig. 1: Coordinate system

With these definitions the plane of the swash plate $\vec{S}_{\text {swash }}$ can be expressed by the Eq. (1), where $\vec{s}_{1}$ and $\vec{s}_{2}$ are the two direction vectors and $\eta$ and $\rho$ are two control variables.

$$
\begin{aligned}
& \vec{S}_{\text {swash }}=\eta \cdot \vec{s}_{1}+\rho \cdot \vec{s}_{2} \\
& \vec{S}_{\text {swash }}=\eta \cdot\left[\begin{array}{l}
1 \\
0 \\
0
\end{array}\right]+\rho \cdot\left[\begin{array}{c}
0 \\
\cos (\alpha) \\
\sin (\alpha)
\end{array}\right]
\end{aligned}
$$

The centre of the ball joint of a piston $\vec{p}_{p}$ is described by Eq. (2), with $\vec{v}_{p}$ as the position vector in the $x_{0}-y_{0}$ plane, $\vec{z}_{p}$ the direction vector of the piston axis and $\lambda$ the associated control variable.

$$
\begin{aligned}
\vec{p}_{p} & =\vec{v}_{p}+\lambda \cdot \vec{z}_{p} \\
\vec{p}_{p} & =\left[\begin{array}{c}
R \cdot \cos (\varphi) \\
R \cdot \sin (\varphi) \\
0
\end{array}\right]+\lambda \cdot\left[\begin{array}{c}
-\cos (\varphi) \cdot \sin (\alpha) \\
-\sin (\varphi) \cdot \sin (\alpha) \\
\cos (\alpha)
\end{array}\right]
\end{aligned}
$$

To get the track of the centres of the ball joints as a function of $\varphi, \alpha$ and $\psi$ the Eq. (1) and Eq. (2) are set equal and the control variables are determined by solving the system of equations, see Eq. (3).

$$
\begin{aligned}
& \eta \cdot \vec{s}_{1}+\rho \cdot \vec{s}_{2}=\vec{v}_{p}+\lambda \cdot \vec{z}_{p} \\
& A \cdot X=B \quad \Rightarrow X=\operatorname{in} v(A) \cdot B \\
& \Rightarrow\left[\begin{array}{c}
\lambda \\
\eta \\
\rho
\end{array}\right]=\operatorname{in} v\left[\begin{array}{lll}
-\vec{z}_{p} & \vec{s}_{1} & \vec{s}_{2}
\end{array}\right] \cdot \vec{v}_{p}
\end{aligned}
$$

One result is the stroke function of the piston $s_{p}$, which is equal to the variable $\lambda$, see Eq. (4). This equation is similar to known equations from ${ }^{6), \text { p.118 }}$ and ${ }^{5), \text { p.20 }}$ with the corresponding parameters.

$$
\lambda=\frac{\mathrm{R} \cdot \sin (\varphi) \cdot \sin (\alpha)}{\cos (\alpha) \cdot \cos (\psi)+\sin (\varphi) \cdot \sin (\alpha) \cdot \sin (\psi)}=s_{p}
$$

In operation mode of the entire unit it is commonly assumed that the ball joint of the uninclined piston is stiffened, so without a degree of freedom, see ${ }^{6), p .170 ; 3)}$. Therefore the pistons rotate with opposite drive speed in the bushing.

To describe the movement, especially the rotation of the inclined pistons, this assumption is partly adopted. But in contrast to the assumption of a completely stiffened joint, a nodding between slipper and piston has to be allowed. For that reason the assumption is changed to a joint with one degree of freedom which allows this necessary nodding.

In Fig. 2 such piston assembly is depicted with the coordinate systems of the piston and the slipper. Using the assumption that both axis $\vec{x}_{s}$ of the slipper and axis $\vec{x}_{p}$ of the piston are in the same direction, the combined axis $\vec{x}_{s p}$ can be calculated by the cross product of the known axis $\vec{z}_{s}$ and $\vec{z}_{p}$, see Eq. (5). In the following diagrams the swash plate angle $\alpha$ is fixed to $15^{\circ}$. All simulated results are computed with MATLAB whereby $\varphi$ is set in with a step width of $2^{\circ}, \alpha$ and $\psi$ with a step width of $0.5^{\circ}$. 


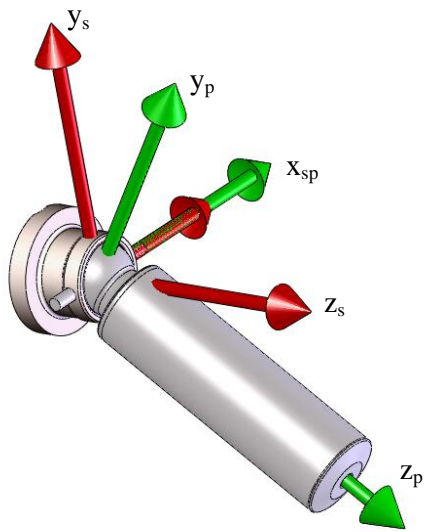

Fig. 2: Ball joint with one degree of freedom

$$
\begin{aligned}
& \vec{z}_{s}=\vec{s}_{1} \times \vec{s}_{2} \\
& \vec{x}_{s p}=\vec{z}_{s} \times \vec{z}_{p} \\
& \vec{p}_{p}=\left[\begin{array}{c}
\sin (\varphi) \cdot \cos (\alpha) \cdot \sin (\psi)-\cos (\psi) \cdot \sin (\alpha) \\
-\cos (\varphi) \cdot \cos (\alpha) \cdot \sin (\psi) \\
-\cos (\varphi) \cdot \sin (\alpha) \cdot \sin (\psi)
\end{array}\right]
\end{aligned}
$$

Figure 3 shows in the plane $\vec{x}_{0}-\vec{y}_{0}$ the direction of the axes $\vec{x}_{s p}(\varphi)$ at an angle ratio $\mu_{\psi \alpha}$ of $50 \%$ and $200 \%$, whereby $\mu_{\psi \alpha}$ is calculated by Eq. (6).

$$
\mu_{\psi \alpha}=\frac{\psi}{\alpha} \cdot 100 \%
$$

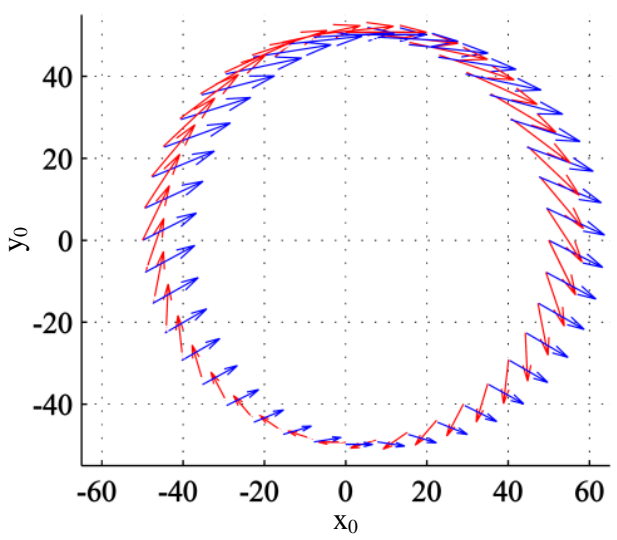

Fig. 3: Direction of $\vec{x}_{s p}$ with $\mu_{\psi \alpha}$ of $50 \%$ (blue) and $200 \%$ (red)

The diagram shows that the direction of the axes at $\mu_{\psi \alpha}=50 \%$ (blue) are only tilting, which means the piston execute a full turn in the rotating bushing, and at $\mu_{\psi \alpha}=200 \%$ (red) the axes execute a full turn, which means the piston is only tilting in the rotating bushing. The angle between $\vec{x}_{s p}(\varphi)$ and axis $\vec{x}_{0}$ is defined as $\vartheta(\varphi)$ by using Eq. (7). The calculation results of the angle $\vartheta(\varphi)$ for different ratios are shown in Figure 4. It can be seen that the rotation positon of an inclined piston leads and lags the uninclined piston position at $\mu_{\psi \alpha}$ less than $100 \%$. Nevertheless, for a full turn of the drive angle $\varphi$, the piston rotates also a full turn in the bushing. $\cos (\vartheta)=\frac{\left|\vec{x}_{s p}(\varphi) \cdot \vec{x}_{0}\right|}{\left|\vec{x}_{s p}(\varphi)\right| \cdot\left|\vec{x}_{0}\right|}$
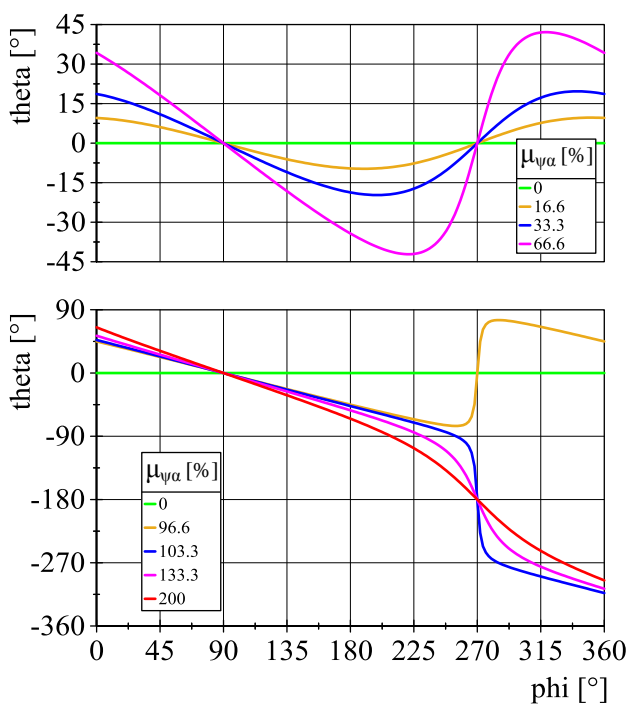

Fig. 4: Angle $\vartheta(\varphi)$ with $\mu_{\psi \alpha}$ from $0 \%$ to $200 \%$

For variable displacement units, it is also possible that the swivel angle $\alpha$ comes below the fixed inclination angle $\psi$. The behaviour of the theoretical angle of the piston is also depicting in Fig. 4. The curves with $\mu_{\psi \alpha}$ above $100 \%$ show that the piston rotates a full turn around its own axis $\vec{z}_{p}$ and therefore the relative rotation in the bushing is in sum zero. In theory, the inner death point at $\varphi=270^{\circ}$ and angle ratio of $\mu_{\psi \alpha}=100 \%$ is discontinuous.

In addition Fig. 5 depicts the angle $\vartheta(\varphi)$ at a constant inclination angle of $5^{\circ}$ and a varying swivel angle of $0^{\circ}$ up to $15^{\circ}$.

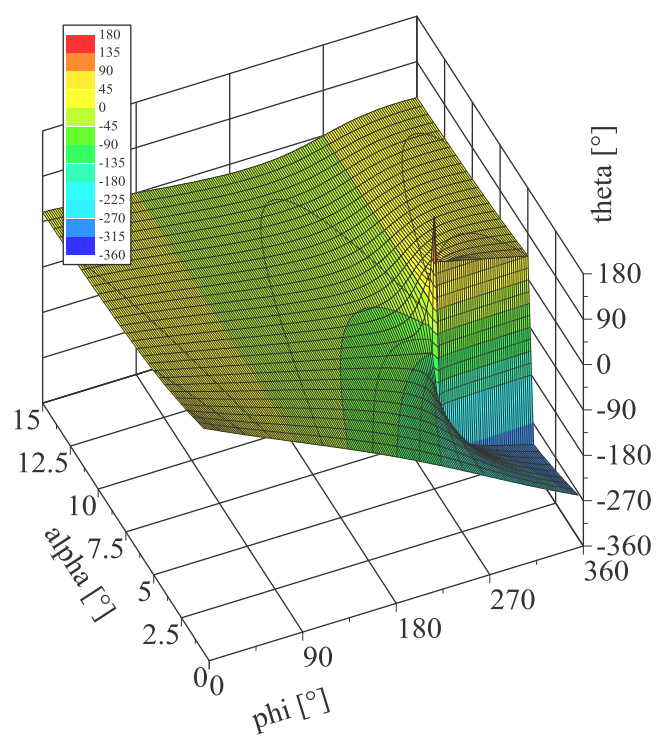

Fig. 5: Angle $\vartheta(\varphi)$ with constant $\psi$ of $5^{\circ}$ and varying swivel angle $\alpha$ 
At a swivel angle of $0^{\circ}$ it is obvious that $\vartheta(\varphi)$ decreases continiously, which means that the piston doesn't execute a relative rotation in the piston. A critical point is at $\alpha=5^{\circ}$ and $\varphi=270^{\circ}$ with an unsteady behaviour due to a very small angle between the rotational axes of the piston and slipper.

For the mechanical losses in the tribological contact the rotational speed is more important. The derivation of Eq. (7) with respect to the time by using the substitution $\varphi \Rightarrow \omega \cdot t$ leads to the rotational speed of the piston around its own axis $\vec{z}_{p}$.

$$
\dot{\vartheta}=\frac{d \vartheta(\varphi)}{d t}=\frac{d \vartheta}{d \varphi} \cdot \frac{d \varphi}{d t}=-\frac{d \vartheta}{d \varphi} \cdot \omega
$$

Figure 6 shows the rotational speed of the piston, with a constant $\alpha=15^{\circ}$ and varying inclination angle, in relation to the drive speed by using Eq. (9).

$\mu_{\dot{\vartheta} \omega}=\frac{\dot{\vartheta}}{\omega} \cdot 100 \%$
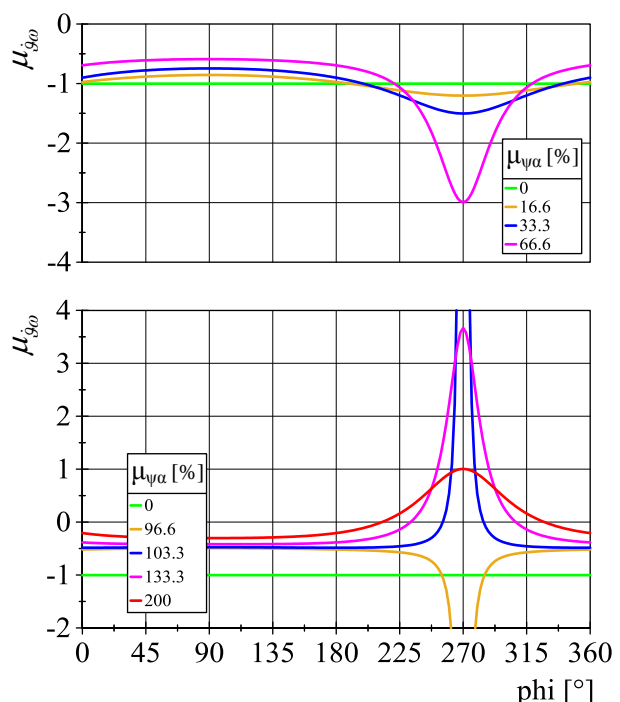

Fig. 6: Rotational speed of the piston in relation to the drive speed

The rotational speed of the piston also leads and lags to the nominal drive speed with contrary direction at $\mu_{\psi \alpha}$ less than $100 \%$. The integral of these curves is "- 1 ". At $\mu_{\psi \alpha}$ greater than $100 \%$ the rotational speed of the piston changes the direction and the integral of these curves are " 0 ", which means that is no relative full turn in this contact.

Additionally, Fig. 7 shows the entire diagram for a $\mu_{\psi \alpha}$ range from $0-200 \%$. In theory the rotation of the inclined piston in the sector around $\varphi=270^{\circ}$ and $\mu_{\psi \alpha} \approx 100 \%$ can obtain a rotational speed which is a few times higher than the drive speed, also with strong accelerations and decelerations around the piston axis.
This approach leads to the assumption that in real applications the piston underlies slippage.

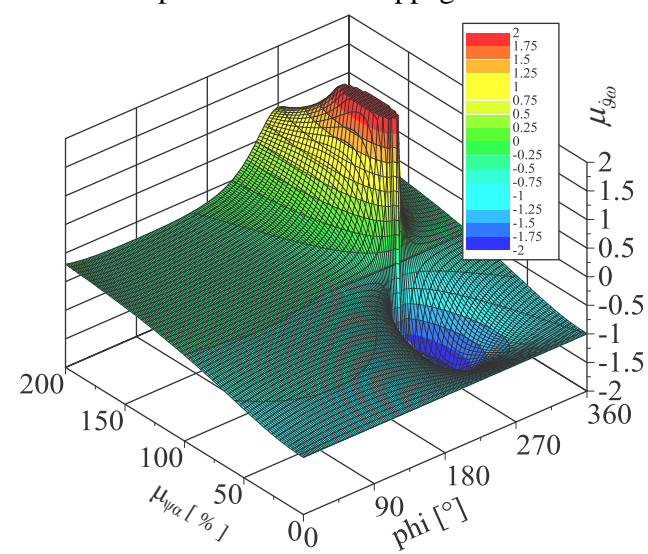

Fig. 7: Rotational speed ratio $\mu_{\dot{\vartheta} \omega}$ with $\mu_{\psi \alpha}$ from $0 \%$ to $200 \%$

To get the total velocity of the piston relative to the bushing, the axial velocity dependent on stroke and tangential velocity based on the rotational speed around the piston axis have to be summarised vectorially.

Table 1 Parameter for exemplary calculation

\begin{tabular}{|c|c|c|c|}
\hline Parameter & Variable & Quantity & Unit \\
\hline Swivel Angle & $\alpha$ & -15 & {$\left[{ }^{\circ}\right]$} \\
\hline Inclination Angle & $\psi$ & 5 & {$\left[{ }^{\circ}\right]$} \\
\hline Piston pitch radius & $R$ & 100 & {$[\mathrm{~mm}]$} \\
\hline Piston diameter & $P$ & 25 & {$[\mathrm{~mm}]$} \\
\hline Drive speed & $n$ & 1500 & {$[\mathrm{rpm}]$} \\
\hline
\end{tabular}

As an example in Table 1 the parameters of a rotation group are defined. The corresponding results are displayed in Fig. 8. It is visible that the total velocity at a rotational angle of $\varphi=270^{\circ}$ achieves its maximum.

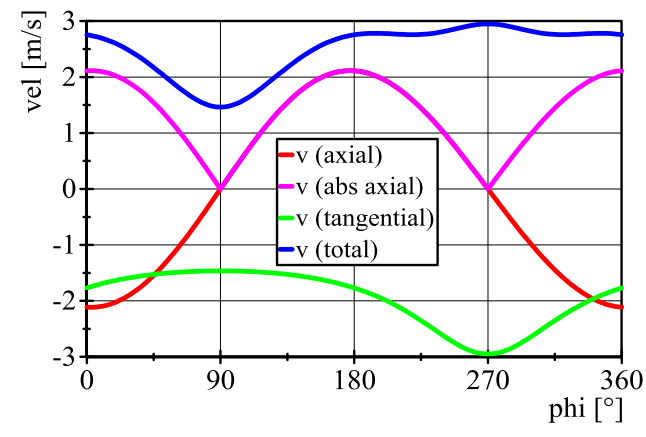

Fig. 8: Velocities of the exemplary unit

It can be concluded that the inclination of the piston could lead to a complex behaviour regarding the rotation and velocity of the piston. The presented equations base on the assumption, that the ball joint between piston and slipper has only one degree of freedom. Therefore, the presented results are considered to be an expectation to compare with measurements. In a real tribological system, the behaviour of 
the joints is affected by friction and resistance, like the inertia of the piston.

\section{Measuring System}

The presented theoretical behaviour of the inclined piston is used for comparison to the actual behaviour by measurements on a single piston test bench, which is similar to the used setup from Breuer ${ }^{5)}$. The kinematics of the swash plate design is inverted to a wobble plate. One piston bushing pair is separated from the cylinder block and fixed in a matrix of piezo-electrical force transducers. A scheme of the test assembly is depicted in Fig. 9 with an inclined bushing carrier. The test bench operates with alternating pressure levels.

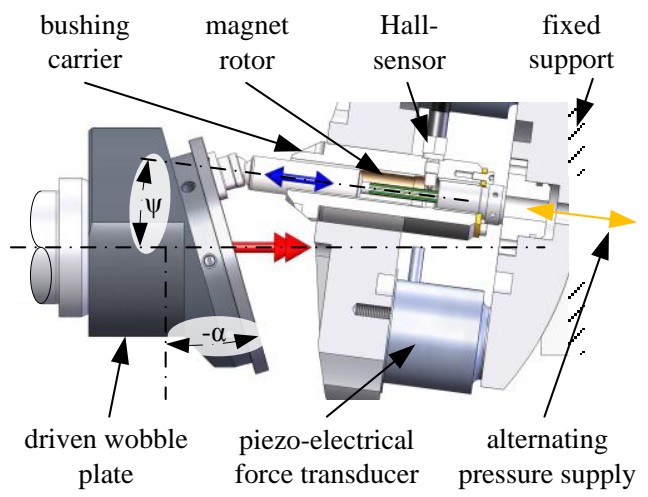

Fig. 9: Scheme of the test set-up; single piston test bench with an inclined piston

Because a measuring device plotting the rotation of the piston is not available on the market, such setup needs to be designed. Some physical effects are suitable to detect the rotation. For example Renius ${ }^{2)}$ used for his measurements of the piston rotation a self-build device whose function principle equalises an electrical generator. A well-known and also commercially used effect was invented by Hall. In automotive applications a combination of such a sensor with a magnetic device is used to detect e.g. the ignition point or the wheel speed. Due to these manifold applications the Hall sensor is a standardised and affordable product.

Because of the piston stroke, the Hall sensor should be fixed while the magnets are placed on a rotor. The fixation of the rotor on the piston is an essential task. The tribological, kinematic and dynamic behaviour of the piston should not be affected. Because of the use of magnets their impact on the piston has to be taken into account. The rotor has to be rotationally symmetric and fixed coaxial to the piston. To accommodate the rotor on the free area of the piston neck provides the benefit that the sensor could be arranged in the low pressurised housing. This arrangement leads to the disadvantage that the sensor has to trail the stroke of the piston. The electrical connection gets higher complexity why this arrangement is not pursued further. Consequently, the rotor is fixed at the pressurised end of the piston and the sensor is screwed in the cylinder block / bushing carrier.

To increase the resolution of the sensor on the rotor five magnet pairs are incorporated. A single magnet has the dimension of $1 \times 0.5 \times 20 \mathrm{~mm}$ (W $\times$ Hx L) and is transversally magnetised with a flux density of 375 mTesla $(=3750 \mathrm{G})$ at a clearance of $1 \mathrm{~mm}$. The rotor is manufactured of aluminium and screwed in an additional threat hole in the piston. The assembled piston with the rotor is shown in Fig. 10.

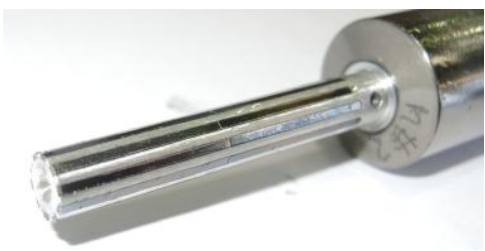

Fig. 10: Assemble magnet rotor at the pistons end

The concentricity of the lateral surface of the rotor is ensured by a later lathe process. Afterwards the grooves for the magnets are wrought and the magnets are glued in. The lateral hole at the end of the rotor ensures the oil supply for the slipper. The weight of the rotor is approximately $8 \mathrm{~g}$.

The Hall sensor is affixed on a pressure resistant holder with three drill holes for the signal wiring, see Fig. 11. The sensor has a dimension of $3 \times 1 \times 2.9 \mathrm{~mm}$ and typical sensitivity of $5 \mathrm{mV} / \mathrm{G}$.

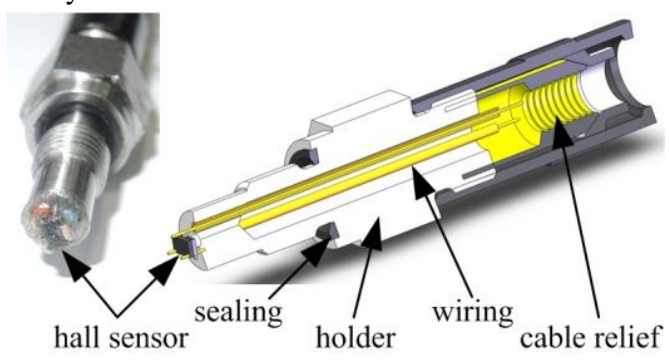

Fig. 11: Installed Hall sensor on the holder

The implementing of the system is done on a lathe at different speeds from $240 \mathrm{rpm}$ up to $2750 \mathrm{rpm}$. A rotary encoder with 5000 increments per revolution was directly connected to the magnet rotor and was used for the reference measurement. The data record is realised by a real time data logger system, using EtherCAT and EtherLab technologie ${ }^{9)}$, with a recording frequency of $20 \mathrm{kHz}$. This system is also used for the measurements on the single piston test bench. 
In the upper diagram of Fig. 12, the unsmoothed raw signal of the Hall sensor at a rotational speed of $2776.22 \mathrm{rpm}$ is depicted for one revolution. The amplitudes of the signal vary due to the unevenly flux density of the magnets and the different clearances between the magnets and the Hall sensor, due to the glueing of the magnets by hand.

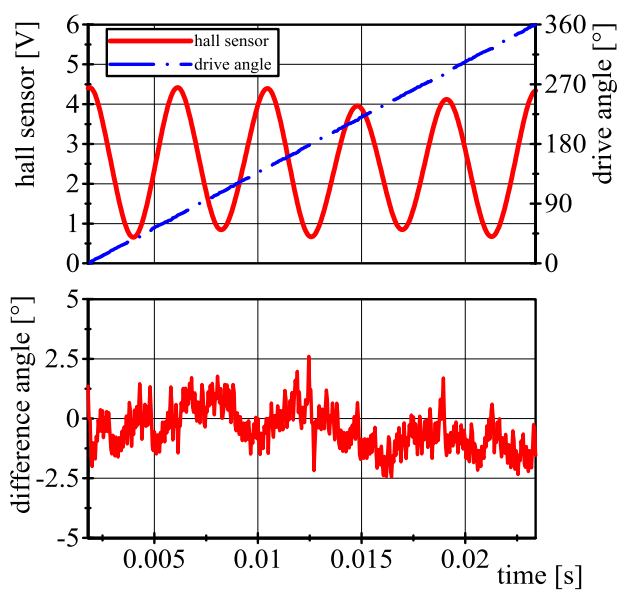

Fig. 12: Raw signal of the Hall sensor and difference angle at $2776 \mathrm{rpm}$

Nevertheless, the calculation of the angle with the Hall sensor signal differs roughly $2.5^{\circ}$, see the lower diagram of Fig. 12. In relation to one revolution a difference angle of $2.5^{\circ}$ leads to a deviation of $0.7 \%$.

A measurement from the test bench, see also Fig. 9, is shown in Fig. 13. At a drive speed of $2750 \mathrm{rpm}$, a high pressure level of 300 bar and an angle ratio $\mu_{\psi \alpha}$ of $18.75 \%$ the sensor signal fits to the expected value of theoretical calculation. For this diagram the inner death point of the piston is at $\varphi=0^{\circ}$.

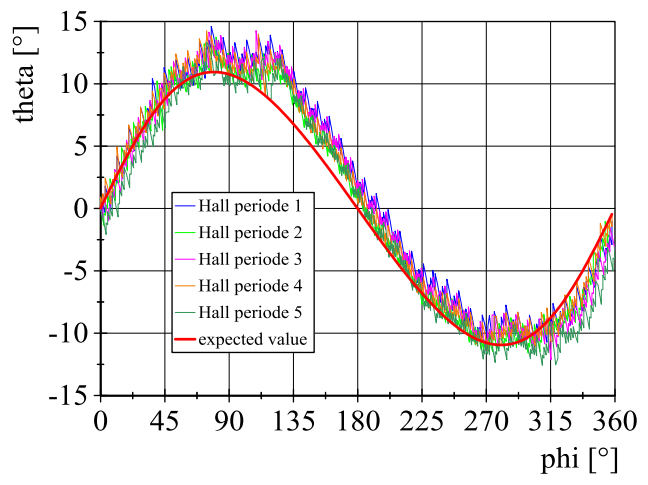

Fig. 13: Measurement from the test bench at $2750 \mathrm{rpm}$ and $300 \mathrm{bar}$ high pressure

\section{Conclusion}

The theoretical analyses of the kinematic of the inclined piston of an axial piston unit in swash plate design shows that the rotation angle and speed depends highly of the ratio between the inclination angle and the swash plate angle. The assembled sensor, utilising the Hall Effect, offers a robust and easy to handle signal. The comparison of the signal of the Hall sensor with the measurement of the high segmented rotary encoder shows a quite good correlation with a deviation of roughly $0.7 \%$. An example measurement from the test bench demonstrates the functionality of the sensor under realistic conditions and validated the rotation of the piston depending of the inclination angle.

Based on the functioning measurement equipment and the theoretical kinematic formulation detailed studies of the piston in the bushing will be conducted.

The gained knowledge will lead to a better understanding of the tribological conditions in the highly stressed pistonbushing contact in axial piston units.

\section{Acknowledgement}

The authors thank the company Bosch Rexroth AG, Germany, for the support and financing of the entire project to investigate the piston bushing contact of swash plate units in detail.

\section{References}

1) Renius, K. Th., Experimentelle Untersuchungen an Gleitschuhen von Axialkolbenmaschinen, Ölhydraulik und Pneumatik 17, 1973, Nr. 3, pp.75-80.

2) Renius, K. Th., Untersuchungen zur Reibung zwischen Kolben und Zylinder bei SchrägscheibenAxialkolbenmaschinen, VDI-Forschungsheft 561, VDIVerlag GmbH, Düsseldorf / Germany, 1974.

3) Fang, Y. and Shirakashi, M., Mixed Lubrication Characteristics Between the Piston und Cylinder in Hydraulic Piston Pump-Motor, Transaction of the ASME, Vol. 117, 1995, pp.80-85.

4) Bräckelmann, U., Reibung, Steifigkeit und Dämpfung in Schrägscheiben-Axialkolbenpumpen und -motoren, PhD-Thesis, ISBN 3-89194-177-3, Bochum / Germany, 2006.

5) Breuer, D., Reibung am Arbeitskolben von Schrägscheibenmaschinen im Langsamlauf; PhD-Thesis; ISBN 978-3-8322-6505-2; Aachen / Germany; 2007.

6) Ivantysyn, J. and Ivantysynova, M., Hydostatic Pumps and Motors, ISBN 81-85522-16-2, New Delhi / India, 2001.

7) IgH Essen, Germany, www.igh-essen.com. 